\title{
a2-antiplasmin modulates bone formation by negatively regulating osteoblast differentiation and function
}

\author{
YOSUKE KANNO $^{1}$, AKIRA ISHISAKI ${ }^{2}$, HIROMI KURETAKE $^{1}$, \\ CHIHIRO MARUYAMA ${ }^{1}$, AYAKA MATSUDA ${ }^{1}$ and OSAMU MATSUO ${ }^{3}$ \\ ${ }^{1}$ Department of Clinical Pathological Biochemistry, Faculty of Pharmaceutical Sciences, Doshisha Women's College \\ of Liberal Arts, Kyoto 610-0395; ${ }^{2}$ Division of Cellular Biosignal Sciences, Department of Biochemistry, \\ Iwate Medical University, Iwate 028-3694; ${ }^{3}$ Kindai University Faculty of Medicine, Osaka 589-8511, Japan
}

Received January 26, 2017; Accepted July 3, 2017

DOI: 10.3892/ijmm.2017.3055

\begin{abstract}
AP})$ is known to be a physiological inhibitor of plasmin. Previously, we showed that $\alpha 2 \mathrm{AP}$ displays various functions, such as promotion of extracellular matrix production, cell growth, and cell differentiation that are not promoted by its function as a plasmin inhibitor. We herein investigated the role of $\alpha 2 \mathrm{AP}$ in bone formation by examining calcein incorporation after its injection in $\alpha 2 \mathrm{AP}$ deficient mice. We found that $\alpha 2 \mathrm{AP}$ deficiency enhanced the bone formation rate in mice. We also found that the osteocalcin expression and alkaline phosphatase activity were elevated in the femur and serum of the $\alpha 2 \mathrm{AP}$-deficient mice. Intriguingly, $\alpha 2$ AP deficiency promoted osteoblast (OB) differentiation of primary calvarial OBs. In contrast, $\alpha 2 \mathrm{AP}$ attenuated $\mathrm{OB}$ differentiation of mouse osteoblastic the MC3T3-E1 cells. Furthermore, $\alpha 2$ AP attenuated Wnt-3a-induced $\beta$-catenin expression and low-density lipoprotein receptor-related protein 6 activation in the MC3T3-E1 cells. These results suggest that $\alpha 2 \mathrm{AP}$ negatively affects $\mathrm{OB}$ differentiation and function by inhibiting the $\mathrm{Wnt} / \beta$-catenin pathway. These findings provide a basis for clinical strategies to improve various bone disorders.
\end{abstract}

\section{Introduction}

Bone homeostasis is regulated by an appropriate balance between resorption of old bone and formation of new bone, which is known as bone remodeling. Osteoblasts (OBs), which are responsible for bone formation, arise from mesenchymal stem cells (MSCs) as OB progenitors, and OB differentiation and function are positively regulated by several signaling pathways including Wnt/ $\beta$-catenin-mediated signaling which

Correspondence to: Dr Yosuke Kanno, Department of Clinical Pathological Biochemistry, Faculty of Pharmaceutical Sciences, Doshisha Women's College of Liberal Arts, 97-1 Kodo, Kyotanabe, Kyoto 610-0395, Japan

E-mail: ykanno@dwc.doshisha.ac.jp

Key words: $\alpha 2$-antiplasmin, osteoblast, bone formation targets the expression of osteogenic transcription factor Runx2 (1,2). Additionally, OBs are associated with differentiation and activation of osteoclasts (OCs) which are responsible for bone resorption (3).

$\alpha 2$-antiplasmin ( $\alpha 2 \mathrm{AP})$ is known to be synthesized in various tissues, and functions as the principal inhibitor of plasmin, a main component of the fibrinolytic system (4,5). As a new function of $\alpha 2 \mathrm{AP}$, we previously found that $\alpha 2 \mathrm{AP}$ is associated with tissue remodeling, angiogenesis, extracellular matrix (ECM) production, cell growth and cell differentiation (6-11). $\alpha 2 \mathrm{AP}$ is most phylogenetically closely related to the non-inhibitory serine protease inhibitor, pigment epithelium-derived factor (PEDF) (12), and they have very similar structure $(3 \beta$-sheets and $9 \beta$-helices) $(13,14)$. Furthermore, Shiomi et al recently reported that $\alpha 2 \mathrm{AP}$ deficiency attenuated ovariectomy (OVX)-induced bone loss, and $\alpha 2 \mathrm{AP}$ is associated with osteoclast formation (15). These observations suggest that $\alpha 2 \mathrm{AP}$ exhibits various functions not only as a plasmin inhibitor, but also as a modulator of bone metabolism. However, the mechanisms underlying $\alpha 2 \mathrm{AP}$-regulated bone metabolism remain to be clarified at the cellular and molecular levels.

We herein investigated the roles of $\alpha 2 \mathrm{AP}$ in bone metabolism, particularly in regards to how $\alpha 2 \mathrm{AP}$ affects $\mathrm{OB}$ differentiation and bone formation.

\section{Materials and methods}

Animals. The $\alpha 2 \mathrm{AP}$-deficient $\left(\alpha 2 \mathrm{AP}^{-/-}\right)$mice were generated by homologous recombination using embryonic stem cells, as previously described (16). Wild-type $\left(\alpha 2 \mathrm{AP}^{+/+}\right)$and $\alpha 2 \mathrm{AP}^{-/-}$mice littermates were housed in groups of $2-5$ in filter-top cages with a fixed 12-h light and 12-h dark cycle.

The animal experiments were approved by the Animal Research Committee of Doshisha Women's College of Liberal Arts (approval ID, Y15-024). All experiments were performed in accordance with relevant guidelines and regulations.

In vivo calcein labeling. Calcein (Nacalai Tesque, Inc., Kyoto, Japan) in saline was intraperitoneally injected into eight-week-old $\alpha 2 \mathrm{AP}^{+/+}$and $\alpha 2 \mathrm{AP}^{-/-}$mice $(20 \mathrm{mg} / \mathrm{kg})$. Two injections were given 3 days apart. The undecalcified sections 
of femurs from eight-week-old $\alpha 2 \mathrm{AP}^{+/+}$and $\alpha 2 \mathrm{AP}^{-/-}$mice were prepared by the Tohkai Cytopathology Institute (Gifu, Japan). Bone formation was visualized using a calcein incorporation assay as described by Naylor et al (17). Briefly, the mineral apposition rate $(\mu \mathrm{m} /$ day) in vivo was calculated by identifying newly formed bone via calcein labeling. The data of double-labeled regions were obtained by using fluorescence microscopy, and the mineral apposition rate was calculated as the distance of the double-labeled regions.

Immunohistochemical staining of osteocalcin. Paraffin-embedded tissue of femurs in eight-week-old $\alpha 2 \mathrm{AP}^{+/+}$ and $\alpha 2 \mathrm{AP}^{-/-}$mice was serially sectioned at 4-7 $\mu \mathrm{m}$ distance. Then, the sections were labeled with anti-rabbit osteocalcin antibody (cat. no. SC-30045; Santa Cruz Biotechnology, Inc., Santa Cruz CA, USA), and then secondarily labeled with Cy3-conjugated anti-rabbit IgG (cat. no. A10520; Thermo Fisher Scientific, Inc., Waltham, MA, USA). The signals were then detected using a laser scanning microscope. The stained images obtained from separate fields on the specimens were analyzed using ImageJ software.

Enzyme-linked immunosorbent assay (ELISA). The osteocalcin in the serum from eight-week-old $\alpha 2 \mathrm{AP}^{+/+}$and $\alpha 2 \mathrm{AP}^{-/-}$mice was then measured using a mouse osteocalcin EIA kit (Biomedical Technologies, Stoughton, MA, USA). The absorbance of the ELISA samples was measured at $450 \mathrm{~nm}$ using Multiskan JX (Thermo LabSystems, Beverly, MA, USA).

Measurement of alkaline phosphatase (ALP) activity. We measured ALP activity in the serum and osteoblasts from $\alpha 2 \mathrm{AP}^{+/+}$and $\alpha 2 \mathrm{AP}^{-/-}$mice as previously described (18). ALP activity was determined using $p$-nitrophenyl phosphate (SigmaAldrich, Steinheim, Germany) as a substrate. The absorbance of the samples was measured at $405 \mathrm{~nm}$ using Multiskan JX (Thermo LabSystems).

Cell culture. Primary $\mathrm{OBs}$ derived from $\alpha 2 \mathrm{AP}^{+/+}$and $\alpha 2 \mathrm{AP}^{-/-}$mouse calvaria were obtained as previously described (19). Primary OBs or MC3T3-E1 cells were maintained in minimum essential medium (MEM) (Invitrogen Life Technologies, Carlsbad, CA, USA) supplemented with $10 \%$ fetal bovine serum (FBS) (Biowest, Nuaillé, France) and 1\% penicillin-streptomycin (Invitrogen Life Technologies) at $37^{\circ}$ in a humidified atmosphere of $5 \% \mathrm{CO}_{2} / 95 \%$ air.

$O B$ differentiation. $\mathrm{OB}$ differentiation in the primary $\mathrm{OBs}$ derived from $\alpha 2 \mathrm{AP}^{+/+}$and $\alpha 2 \mathrm{AP}^{-/-}$mouse calvaria and MC3T3-E1 cells were induced as previously described (19). Briefly, primarily cultured OBs or MC3T3-E1 cells were cultured for 14 days in the differentiation media supplemented with $10 \mathrm{mM} \beta$-glycerophosphate and $10 \mathrm{nM}$ dexamethasone (both from Sigma-Aldrich), and $50 \mu \mathrm{g} / \mathrm{ml}$ ascorbic acid (Wako Pure Chemical Industries, Ltd., Osaka, Japan) in 6-well plates. After 14 days, the cells were then washed with phosphatebuffered saline (PBS), and cell proteins were extracted with a lysis buffer (10 mM Tris-HCl, $\mathrm{pH} 7.5,0.1 \%$ Triton X-100).

Reverse transcription-polymerase chain reaction (RT-PCR). We performed RT-PCR as previously described (19).
First-strand cDNA was synthesized from total RNA using the High Fidelity RT-PCR kit (Toyobo, Osaka, Japan). Quantitative RT-PCR (RT-qPCR) was performed on the IQ5 real-time PCR detection system (Bio-Rad Laboratories, Inc., Hercules, CA, USA) with SYBR-Green technology on cDNA generated from the reverse transcription of purified RNA. The 2-step PCR reactions were performed as $92^{\circ} \mathrm{C}$ for $1 \mathrm{sec}$ and $60^{\circ} \mathrm{C}$ for $10 \mathrm{sec}$. Runx 2 mRNA expression was normalized against GAPDH mRNA expression using the comparative cycle threshold method. We used the following primer sequences: Runx2 forward, 5'-GAATGGCAGCACGCTATTAAATCC-3' and reverse, 5'-GCCGCTAGAATTCAAAACAGTTGG-3'; GAPDH forward, 5'-TTCATTGACCTCAACTACATG-3' and reverse, 5'-GTGGCAGTGATGGCATGGAC-3'.

Western blot analysis. Western blot analysis was performed as previously described (20). Briefly, cells were washed twice with cold PBS, harvested, and then sonicated in lysis buffer containing $10 \mathrm{mM}$ Tris-HCl buffer (pH 7.5), 1\% SDS, $1 \%$ Triton X-100, and a protease inhibitor cocktail (Roche, Mannheim, Germany). The protein concentration in each lysate was measured using a BCA protein assay kit (Pierce, Rockford, IL, USA). Proteins in the supernatant were separated by electrophoresis on $10 \%$ SDS-polyacrylamide gels and transferred to a PVDF membrane. We detected $\beta$-catenin, phospho-lipoprotein receptor-related protein 6 (p-LRP6), LRP6 and GAPDH by incubation with anti-rabbit $\beta$-catenin antibody (cat. no. Rb-1491; NeoMarkers, Fremont, CA, USA), anti-rabbit phospho-LRP6 antibody (cat. no. bs-2905R) and anti-rabbit LRP6 antibody (cat. no. bs-3253R) (both from Bioss Inc., Woburn, MA, USA), and anti-rabbit GAPDH antibody (cat. no. SAB2100894; Sigma-Aldrich) followed by incubation with horseradish peroxidase-conjugated antibodies to rabbit IgG (cat. no. NA934-1ML; Amersham Pharmacia Biotech, Uppsala, Sweden).

Spontaneous secretion of VEGF in primary OBs. Spontaneous secretion of VEGF in primary OBs was measured as previously described (6). The OBs were maintained in MEM $\alpha$ containing $10 \%$ FBS. After 6 days, the medium was exchanged for serum-free MEM $\alpha$. After $24 \mathrm{~h}$, the conditioned medium was collected, and VEGF in the medium was then measured by VEGF ELISA kit (R\&D Systems, Minneapolis, MN, USA). The absorbance of the ELISA samples was measured at $450 \mathrm{~nm}$ using Multiskan JX (Thermo LabSystems).

Statistical analysis. All data are expressed as the means \pm SEM. The significance of the effect of each treatment $(\mathrm{P}<0.05)$ was determined by analysis of variance (ANOVA) followed by the least significant difference test.

\section{Results}

Effect of $\alpha 2 A P$ deficiency on bone formation in mice. We investigated the effect of $\alpha 2 \mathrm{AP}$ deficiency on bone formation by examining calcein incorporation after its injection in the $\alpha 2 \mathrm{AP}^{+/+}$and $\alpha 2 \mathrm{AP}^{-/-}$mice. The double calcein-labeled regions in the femurs from the eight-week-old $\alpha 2 \mathrm{AP}^{+/+}$ and $\alpha 2 \mathrm{AP}^{-/-}$mice are shown in Fig. 1A. The mineral apposition rate was calculated as the distance of the double calcein-labeled 
A Calcein

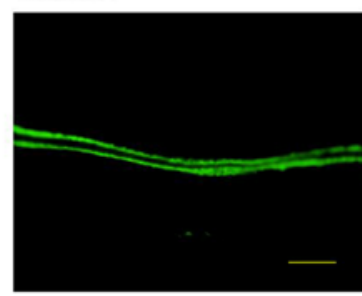

$\alpha 2 \mathrm{AP}^{+1+}$

Osteocalcin

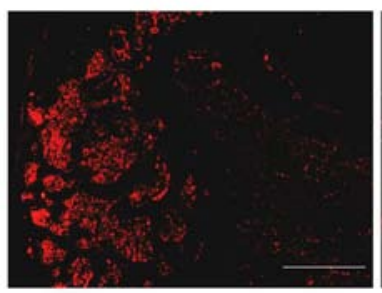

$\alpha 2 \mathrm{AP}^{+/+}$

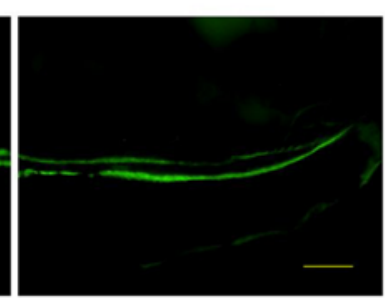

$\alpha 2 \mathrm{AP}-/$

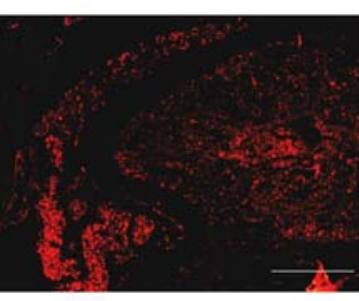

$\alpha 2 A P \%$
B

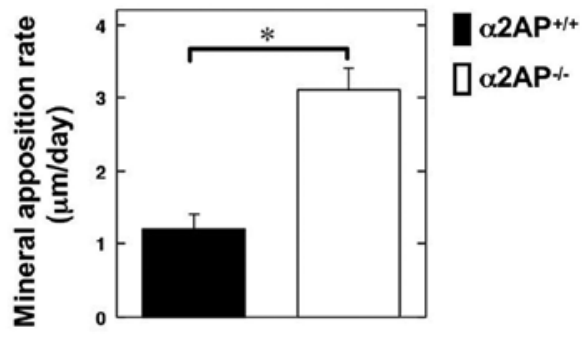

D

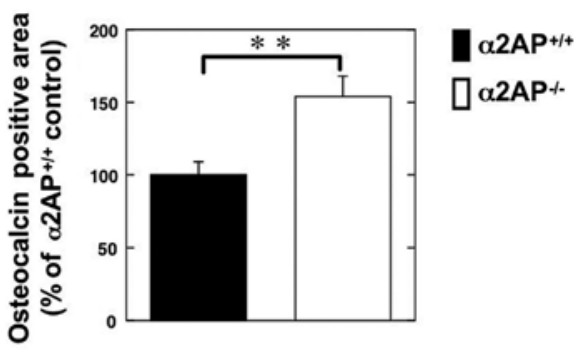

F

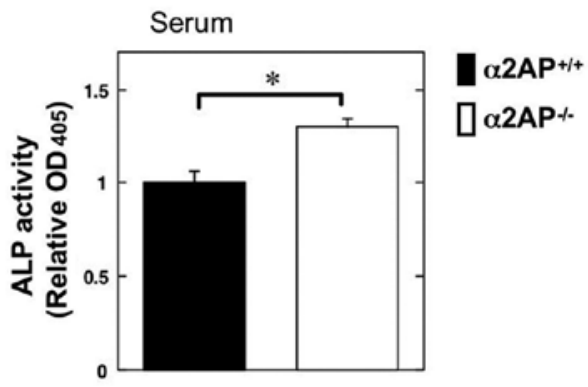

Figure 1. Effect of $\alpha 2$-antiplasmin ( $\alpha 2 \mathrm{AP}$ ) deficiency on bone formation in mice. (A) Eight-week-old $\alpha 2 \mathrm{AP}^{+/+}$and $\alpha 2 \mathrm{AP}^{-/-}$mice were given 2 injections of calcein 3 days apart to label mineralizing bone surfaces in the femur. Scale bar, $100 \mu \mathrm{m}$. (B) The distance between these labels, measured in the trabecular bone at the epiphysis of femur sections, was determined as the mineral apposition rate $(\mathrm{n}=6)$. (C) The osteocalcin staining (red) of tissue section of femurs in eight-week-old $2 \mathrm{AP}^{+/+}$and $\alpha 2 \mathrm{AP}^{-/-}$mice was performed as described in Materials and methods. Scale bar, $200 \mu \mathrm{m}$. (D) The intensity of osteocalcin expression in the tissue sections was quantitatively evaluated as described in Materials and methods (n=4). (E) The quantitative level of osteocalcin in serum from the $\alpha 2 \mathrm{AP}^{+/+}$and $\alpha 2 \mathrm{AP}^{-/}$mice was evaluated as described in Materials and methods $(\mathrm{n}=4)$. (F) The alkaline phosphatase (ALP) activity in serum from the $\alpha 2 \mathrm{AP} /++$ and $\alpha 2 \mathrm{AP}^{-/-}$mice was evaluated as described in Materials and methods $(\mathrm{n}=4)$. The data represent the mean $\pm \mathrm{SEM}$. ${ }^{*} \mathrm{P}<0.01 ;{ }^{* *} \mathrm{P}<0.05$.

regions. The distance between the double calcein-labels in the femurs from the $\alpha 2 \mathrm{AP}^{-/-}$mice was larger than that from the $\alpha 2 \mathrm{AP}^{+/+}$mice (Fig. 1B). Additionally, we examined the expression of osteocalcin in the femurs from the $\alpha 2 \mathrm{AP}^{+/+}$ and $\alpha 2 \mathrm{AP}^{-/-}$mice. The level of osteocalcin expression in the femurs from the $\alpha 2 \mathrm{AP}^{-/-}$mice was significantly higher than that of the $\alpha 2 \mathrm{AP}^{+/+}$mice at the protein level (Fig. 1C and D). Furthermore, we examined the levels of osteocalcin and ALP activity in the serum of the $\alpha 2 \mathrm{AP}^{+/+}$and $\alpha 2 \mathrm{AP}^{-/-}$mice. The levels of osteocalcin and ALP activity in the serum of the $\alpha 2 \mathrm{AP}^{-/-}$mice were significantly higher than those of the $\alpha 2 \mathrm{AP}^{+/+}$mice (Fig. 1E and F, respectively).

Effect of $\alpha 2 A P$ deficiency on $O B$ differentiation and function. Next, to clarify the role of $\alpha 2 \mathrm{AP}$ in $\mathrm{OB}$ differentiation, we examined the ALP activity in primary calvarial OBs from the $\alpha 2 \mathrm{AP}^{+/+}$and $\alpha 2 \mathrm{AP}^{-/-}$mice in the absence or presence of $\mathrm{OB}$ differentiation media. Intriguingly, the $\alpha 2 \mathrm{AP}$ deficiency resulted in upregulation of ALP activity in OBs (Fig. 2A).
Additionally, we examined the expression level of Runx2, which is an essential transcription factor for OB differentiation, in $\mathrm{OBs}$ from the $\alpha 2 \mathrm{AP}^{+/+}$and $\alpha 2 \mathrm{AP}^{-/-}$mice in the presence of the OB differentiation media. The level of Runx2 mRNA expression in the $\alpha 2 \mathrm{AP}^{-/-}$OBs was significantly higher than that in the WT OBs (Fig. 2B). It has been reported that PEDF, which is most phylogenetically closely related to $\alpha 2 \mathrm{AP}$, inhibits the Wnt/ $\beta$-catenin pathway by blocking LRP6 (21). Therefore, to clarify whether or not $\alpha 2 \mathrm{AP}$ is associated with the Wnt $\beta$ catenin pathway, we examined the expression of $\beta$-catenin in $\mathrm{OBs}$ from the $\alpha 2 \mathrm{AP}^{+/+}$and $\alpha 2 \mathrm{AP}^{-/-}$mice. The expression of $\beta$-catenin in the $\alpha 2 \mathrm{AP}^{-/-} \mathrm{OBs}$ was significantly higher than that in the $\alpha 2 \mathrm{AP}^{+/+} \mathrm{OBs}$ at the protein level (Fig. 2C).

Effect of $\alpha 2 A P$ on $O B$ differentiation and function. In order to clarify the roles of $\alpha 2 \mathrm{AP}$ in the functions of $\mathrm{OBs}$, we examined ALP activity in the $\alpha 2 \mathrm{AP}$-treated mouse osteoblastic MC3T3-E1 cells. $\alpha 2 \mathrm{AP}$ treatment attenuated the OB differentiation media-induced ALP activation 
A

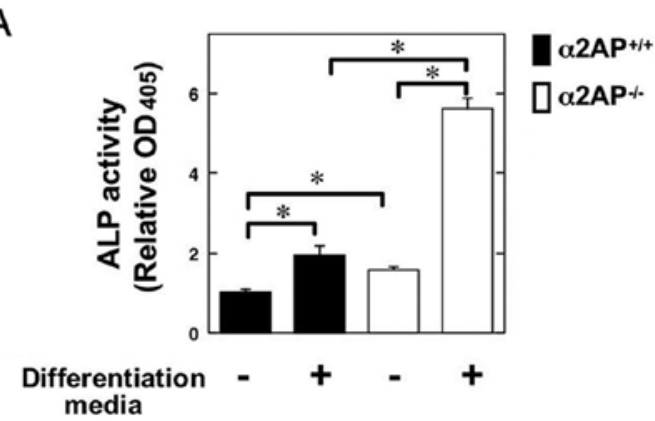

B

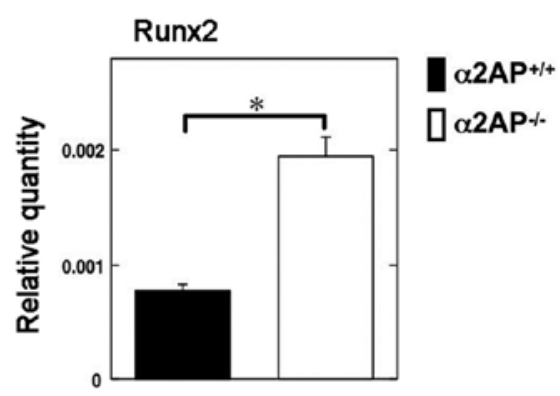

C
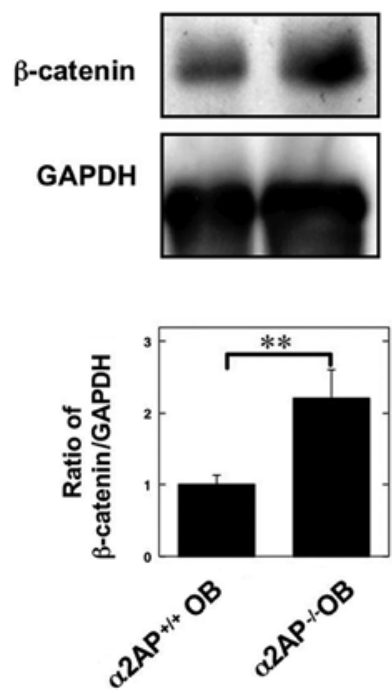

Figure 2. Effect of $\alpha 2$-antiplasmin ( $\alpha 2 \mathrm{AP}$ ) deficiency on osteoblast (OB) differentiation and function. (A and $\mathrm{B}$ ) $\mathrm{OBs}$ from the $\alpha 2 \mathrm{AP}^{+/+}$and $\alpha 2 \mathrm{AP}^{-/ /}$mice were cultured for 14 days in the absence or presence of differentiation media. (A) Alkaline phosphatase (ALP) activity in $\mathrm{OBs}$ from the $\alpha 2 \mathrm{AP}^{+/+}$ and $\alpha 2 \mathrm{AP}^{-/}$mice was evaluated as described in Materials and methods $(\mathrm{n}=3)$. (B) Expression of Run $x 2 \mathrm{mRNA}$ in OBs from the $\alpha 2 \mathrm{AP}^{+/+}$and $\alpha 2 \mathrm{AP}^{-/-}$mice in the presence of differentiation media was evaluated as described in Materials and methods $(\mathrm{n}=3)$. (C) Expression of $\beta$-catenin in OBs from the $\alpha 2 \mathrm{AP}^{+/+}$ and $\alpha 2 \mathrm{AP}^{-/ /}$mice was evaluated by a western blot analysis. The histogram (bottom panel) shows quantitative representations of $\beta$-catenin obtained from densitometry analysis after normalization to the levels of GAPDH expression $(\mathrm{n}=3)$. The data represent the mean \pm SEM. ${ }^{*} \mathrm{P}<0.01 ;{ }^{* *} \mathrm{P}<0.05$.

in the MC3T3-E1 cells (Fig. 3A). We also showed that $\alpha 2 \mathrm{AP}$ treatment significantly attenuated the Runx2 mRNA expression in the MC3T3-E1 cells in the OB differentiation media (Fig. 3B). Furthermore, we demonstrated that $\alpha 2 \mathrm{AP}$ attenuated the Wnt-3a-induced $\beta$-catenin expression and LRP6 phosphorylation at the protein level (Fig. 3C).

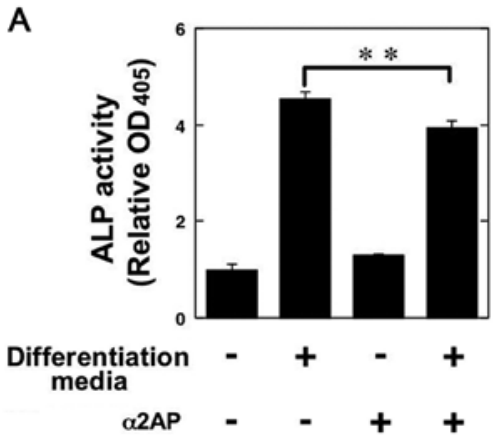

B

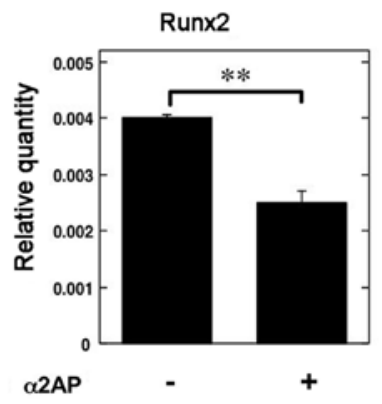

C
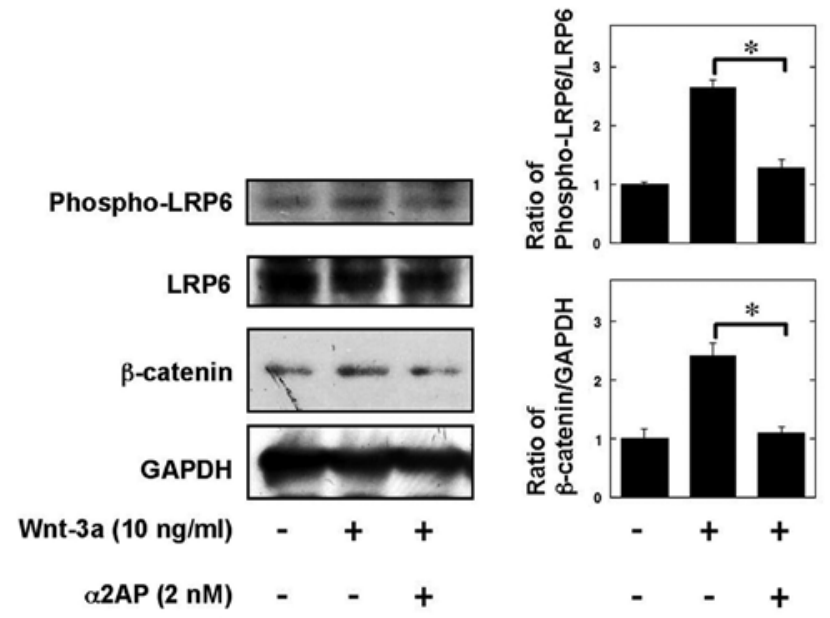

Figure 3. Effect of $\alpha 2$-antiplasmin ( $\alpha 2 \mathrm{AP}$ ) on osteoblast $(\mathrm{OB})$ differentiation and function. (A and B) MC3T3-E1 cells were cultured for 14 days in the absence or presence of differentiation media or $\alpha 2 \mathrm{AP}(1 \mathrm{nM})$ as indicated. (A) Alkaline phosphatase (ALP) activity in MC3T3-E1 cells was evaluated as described in Materials and methods $(\mathrm{n}=3)$. (B) Expression of Runx2 mRNA in MC3T3-E1 cells in the presence of differentiation media with or without $\alpha 2 \mathrm{AP}(1 \mathrm{nM})$ was evaluated as described in Materials and methods $(\mathrm{n}=3)$. (C) MC3T3-E1 cells were cultured for $2 \mathrm{~h}$ in the absence or presence of Wnt-3a $(10 \mathrm{ng} / \mathrm{ml})$ or $\alpha 2 \mathrm{AP}(2 \mathrm{nM})$ as indicated. Then, phosphorylation status of lipoprotein receptor-related protein 6 (LRP6) and $\beta$-catenin expression were examined by a western blot analysis. The histograms (right panels) show quantitative representations of phospho-LRP6 and $\beta$-catenin obtained from densitometry analysis after normalization to the levels of total LRP6 and GAPDH expression, respectively $(n=3)$. The data represent the mean \pm SEM. ${ }^{*} \mathrm{P}<0.01 ;{ }^{* *} \mathrm{P}<0.05$.

\section{Discussion}

In the present study, we investigated the roles of $\alpha 2 \mathrm{AP}$ in the statuses of differentiation and function of OBs that regulate bone formation. We found that the bone formation rate and osteocalcin expression in the femur and serum ALP activity were significantly 
elevated in the $\alpha 2 \mathrm{AP}$-deficient mice compared with these parameters in the WT mice (Fig. 1). Additionally, $\alpha 2 \mathrm{AP}$ deficiency promoted osteogenic transcription factor expression and ALP activity in OBs (Fig. 2A and B). In contrast, the $\alpha 2 \mathrm{AP}$ treatment attenuated them (Fig. 3A and B). These data strongly suggest that $\alpha 2 \mathrm{AP}$ negatively regulates $\mathrm{OB}$ differentiation and function.

Although $\alpha 2 \mathrm{AP}$ is known to be a plasmin inhibitor, we previously found that $\alpha 2 \mathrm{AP}$ regulates ECM production, cell growth, and cell differentiation in the absence of plasmin (7-10). We also showed that plasminogen deficiency did not affect OB differentiation (19). We herein showed that $\alpha 2 \mathrm{AP}$ attenuated OB differentiation in the absence of plasmin (Fig. 3A and B). These observations suggest that $\alpha 2 \mathrm{AP}$-mediated OB differentiation is not carried out by its action as a plasmin inhibitor.

$\alpha 2 \mathrm{AP}$ is most phylogenetically closely related to PEDF (12), and they have very similar structure ( $3 \beta$-sheets and $9 \alpha$-helices) $(13,14)$. It has been reported that PEDF inhibits the Wnt//-catenin pathway by blocking LRP6, which is coreceptor for Wnts (21). The Wnt-3a-induced LRP6 activation results in inhibition of $\beta$-catenin degradation (22), and the Wnt/LRP6/ $\beta$-catenin axis plays an important role in OB differentiation (23-25). We herein showed that the expression status of $\beta$-catenin was elevated in the OBs from the $\alpha 2 \mathrm{AP}$ deficient mice than in that from WT mice (Fig. 2C). In addition, the $\alpha 2 \mathrm{AP}$ treatment attenuated Wnt-3a-induced $\beta$-catenin expression and LRP6 activation (Fig. 3C). These data strongly suggest that $\alpha 2 \mathrm{AP}$ negatively modulates $\mathrm{OB}$ differentiation by inhibiting the Wnt/LRP6//-catenin axis.

In a previous study, we showed that $\alpha 2 \mathrm{AP}$ deficiency enhanced VEGF expression in fibroblasts (6). $\alpha 2 \mathrm{AP}$ deficiency also enhanced VEGF expression in primary osteoblasts (data not shown). It has been reported that osteoblast-derived VEGF positively regulates $\mathrm{OB}$ differentiation and bone formation activity of OBs in autocrine or paracrine manners (26). Additionally, activation of the Wnt/ $\beta$-catenin pathway induces VEGF production (27). The $\alpha 2 \mathrm{AP}$-mediated $\mathrm{Wnt} / \beta$-catenin pathway may also regulate the production of VEGF production, and the $\alpha 2 \mathrm{AP}-$ regulated VEGF production may be associated with bone homeostasis.

In conclusion, $\alpha 2 \mathrm{AP}$ affects bone metabolism by negatively regulating $\mathrm{OB}$ differentiation and function. These findings provide a basis for therapeutic strategies for various bone disorders.

\section{References}

1. Buttery LD, Bourne S, Xynos JD, Wood H, Hughes FJ Hughes SP, Episkopou V and Polak JM: Differentiation of osteoblasts and in vitro bone formation from murine embryonic stem cells. Tissue Eng 7: 89-99, 2001.

2. Yavropoulou MP and Yovos JG: The role of the Wnt signaling pathway in osteoblast commitment and differentiation. Hormones (Athens) 6: 279-294, 2007.

3. Long F: Building strong bones: molecular regulation of the osteoblast lineage. Nat Rev Mol Cell Biol 13: 27-38, 2011.

4. Collen D: Identification and some properties of a new fast-reacting plasmin inhibitor in human plasma. Eur J Biochem 69:209-216, 1976.

5. Menoud PA, Sappino N, Boudal-Khoshbeen M, Vassalli JD and Sappino AP: The kidney is a major site of alpha(2)-antiplasmin production. J Clin Invest 97: 2478-2484, 1996.

6. Kanno Y, Hirade K, Ishisaki A, Nakajima K, Suga H, Into T, Matsushita K, Okada K, Matsuo O and Matsuno H: Lack of alpha2-antiplasmin improves cutaneous wound healing via over-released vascular endothelial growth factor-induced angiogenesis in wound lesions. J Thromb Haemost 4: 1602-1610, 2006.
7. Kanno Y, Kuroki A, Okada K, Tomogane K, Ueshima S, Matsuo $\mathrm{O}$ and Matsuno $\mathrm{H}$ : Alpha2-antiplasmin is involved in the production of transforming growth factor betal and fibrosis. $\mathbf{J}$ Thromb Haemost 5: 2266-2273, 2007.

8. Kanno Y, Kawashita E, Minamida M, Kaneiwa A, Okada K, Ueshima S, Matsuo O and Matsuno H: Alpha2-antiplasmin is associated with the progression of fibrosis. Am J Pathol 176: 238-245, 2010.

9. Kanno Y, Kawashita E, Kokado A, Kuretake H, Ikeda K, Okada K, Seishima M, Ueshima S, Matsuo O and Matsuno H: a2AP mediated myofibroblast formation and the development of renal fibrosis in unilateral ureteral obstruction. Sci Rep 4: 5967, 2014.

10. Kawashita E, Kanno Y, Asayama H, Okada K, Ueshima S, Matsuo $\mathrm{O}$ and Matsuno $\mathrm{H}$ : Involvement of $\alpha 2$-antiplasmin in dendritic growth of hippocampal neurons. J Neurochem 126: 58-69, 2013.

11. Kanno Y, Kawashita E, Kokado A, Okada K, Ueshima S, Matsuo O and Matsuno H: Alpha2-antiplasmin regulates the development of dermal fibrosis in mice by prostaglandin $\mathrm{F}(2 \alpha)$ synthesis through adipose triglyceride lipase/calcium-independent phospholipase A(2). Arthritis Rheum 65: 492-502, 2013.

12. Irving JA, Pike RN, Lesk AM and Whisstock JC: Phylogeny of the serpin superfamily: implications of patterns of amino acid conservation for structure and function. Genome Res 10: 1845-1864, 2000.

13. Law RH, Sofian T, Kan WT, Horvath AJ, Hitchen CR, Langendorf CG, Buckle AM, Whisstock JC and Coughlin PB: X-ray crystal structure of the fibrinolysis inhibitor alpha2-antiplasmin. Blood 111: 2049-2052, 2008.

14. Tombran-Tink J, Aparicio S, Xu X, Tink AR, Lara N, Sawant S, Barnstable CJ and Zhang SS: PEDF and the serpins: phylogeny, sequence conservation, and functional domains. J Struct Biol 151: 130-150, 2005.

15. Shiomi A, Kawao N, Yano M, Okada K, Tamura Y, Okumoto K, Matsuo O, Akagi M and Kaji $\mathrm{H}$ : $\alpha 2$-Antiplasmin is involved in bone loss induced by ovariectomy in mice. Bone 79: 233-241, 2015.

16. Okada K, Lijnen HR, Dewerchin M, Belayew A, Matsuo O, Collen D and Bernaerts R: Characterization and targeting of the murine alpha2-antiplasmin gene. Thromb Haemost 78: 1104-1110, 1997.

17. Naylor AJ, Azzam E, Smith S, Croft A, Poyser C, Duffield JS, Huso DL, Gay S, Ospelt C, Cooper MS, et al: The mesenchymal stem cell marker CD248 (endosialin) is a negative regulator of bone formation in mice. Arthritis Rheum 64: 3334-3343, 2012.

18. Kanno Y, Into T, Lowenstein CJ and Matsushita K: Nitric oxide regulates vascular calcification by interfering with TGF-signalling. Cardiovasc Res 77: 221-230, 2008.

19. Kanno Y, Ishisaki A, Kawashita E, Chosa N, Nakajima K, Nishihara T, Toyoshima K, Okada K, Ueshima S, Matsushita K, et al: Plasminogen/plasmin modulates bone metabolism by regulating the osteoblast and osteoclast function. J Biol Chem 286: 8952-8960, 2011.

20. Kanno Y, Shu E, Kanoh H and Seishima M: The antifibrotic effect of $\alpha 2$ AP neutralization in systemic sclerosis dermal fibroblasts and mouse models of systemic sclerosis. J Invest Dermatol 136: 762-769, 2016.

21. Park K, Lee K, Zhang B, Zhou T, He X, Gao G, Murray AR and Ma JX: Identification of a novel inhibitor of the canonical Wnt pathway. Mol Cell Biol 31: 3038-3051, 2011.

22. Tamai K, Zeng X, Liu C, Zhang X, Harada Y, Chang Z and He X: A mechanism for Wnt coreceptor activation. Mol Cell 13: 149-156, 2004.

23. Wang J, Guan X, Guo F, Zhou J, Chang A, Sun B, Cai Y, Ma Z, Dai C, Li X, et al: miR-30e reciprocally regulates the differentiation of adipocytes and osteoblasts by directly targeting low-density lipoprotein receptor-related protein 6. Cell Death Dis 4: e845, 2013.

24. Fei Y, Xiao L, Doetschman T, Coffin DJ and Hurley MM: Fibroblast growth factor 2 stimulation of osteoblast differentiation and bone formation is mediated by modulation of the Wnt signaling pathway. J Biol Chem 286: 40575-40583, 2011.

25. Honda T, Yamamoto H, Ishii A and Inui M: PDZRN3 negatively regulates BMP-2-induced osteoblast differentiation through inhibition of Wnt signaling. Mol Biol Cell 21: 3269-3277, 2010.

26. $\mathrm{Hu} \mathrm{K}$ and Olsen BR: Osteoblast-derived VEGF regulates osteoblast differentiation and bone formation during bone repair. J Clin Invest 126: 509-526, 2016.

27. Wang Y, Sang A, Zhu M, Zhang G, Guan H, Ji M and Chen H: Tissue factor induces VEGF expression via activation of the Wnt $/ \beta$-catenin signaling pathway in ARPE-19 cells. Mol Vis 22: 886-897, 2016. 